\title{
Abundance and distribution of sessile invertebrates under intertidal boulders (São Paulo, Brazil)
}

\author{
Rosana Moreira da ROCHA*
}

Departamento de Zoologia, Setor de Ciências Biológicas, Universidade Federal do Paraná (Caixa Postal 19020, 81531-990, Curitiba, PR, Brasil)

- Abstract: The encrusting communities under two boulder fields (Praia Grande and Ponta do Balceiro) were monitored monthly during 1990 and 1991, in São Sebastião, on the northern coast of São Paulo State, Brazil. Two sizes of boulders were chosen: small $\left(20-30 \mathrm{~cm}^{2}\right.$ underside area) and larger ones $\left(160-220 \mathrm{~cm}^{2}\right)$ located on the middle and lower levels of the intertidal. The community's components were mainly sessile animals either compound ones such as Bryozoa, Ascidiacea, Porifera and Cnidaria, in this order of abundance, or simple ones such as Polychacta and Bivalvia, also in this order of abundance. All groups, except by serpulids (Polychacta), had higher percent cover in the low intertidal region and under large boulders. Diversity was higher at Ponta do Baleciro, and in the low intertidal region and on large boulders for both shores.

- Resumo: Em São Scbastiāo, litoral norte do Estado de São Paulo, Brasil, foram monitorados mensalmente dois ambientes de matações em costôes rochosos, Praia Grande e Ponta do Baleeiro, ao longo de 1990 e 1991. As condiçōes ambientais avaliadas foram: temperatura e salinidade da água, hidrodinâmica, capacidade de abrasão da areia acumulada, heterogeneidade ambiental e porosidade das pedras. Foi estudąda a comunidade incrustante na superfície inferjor de pedras pequenas $\left(20-30 \mathrm{~cm}^{2}\right.$ de área na face inferior) e maiores $\left(160-220 \mathrm{~cm}^{2}\right)$ dispostas nos estratos médio e inferior da zona entremarés. Esta comunidade era constituída principalmente por organismos sésseis coloniais (Bryozoa, Ascidiacea, Porifera e Cnidaria, nesta ordem de abundância) ou solitários (Polychaeta e Bivalvia, nesta ordem de abundância). Todos os grupos, com exceção dos serpulídeos (Polychaeta), apresentaram maior porcentagem de cobertura nos estratos inferiores e nas pedras grandes. A composiçao específica foi similar nos dois costōes estudados, mas várias espécies ocorreram exclusivamente em um determinado nível de maré, ou tamanho de pedra, indicando que estes fatores têm maior influência na distribuiçāo das espécies. A diversidade foi maior na Ponta do Baleeiro, nos estratos inferiores e em pedras grandes, nos dois costôes, não havendo nenhum tipo de padrâo sazonal de variaçāo.

- Descriptors: Encrusting community, Boulders, Diversity, Intertidal, Bryozoa, Ascidiacea, Porifera, Serpulidae, Sâo Sebastiâo Channel, Sâo Paulo, Brazil.

- Descritores: Comunidade incrustante, Matações, Região Entremarés, Diversidade, Bryozoa, Ascidiacea, Porifera, Serpulidae, Canal de São Sebastiâo, São Paulo, Brasil.

\section{Introduction}

Most work describing the distribution of encrusting communities in the intertidal region deal with stabilized hard substrate, such as vertical walls or large boulders.
Small boulders, on the other hand, are non-stabilized substrata as they suffer displacement and overturning, therefore subjecting encrusting organisms to abrasion, smothering or over-exposition to sunlight and predators.

The exposed upper surfaces of small boulders are usually covered by algae (Sousa, 1979a, b; Littler \& Litter, 1981, 1984; McGuinness \& Underwood, 1986) while the 
undersurfaces are covered by sessile or sedentary animals (Osman, 1977; McGuinness, 1987a, b).

The distribution of the fauna on stabilized substrata is fairly known for many shores in Sāo Paulo State, and we find reports on both the vertical distribution (Nonato \& Pérès, 1961; Oliveira- Filho \& Mayal, 1976; Petersen et al., 1986; Kadekaru et al., 1987; Paula, 1987; Rosso, 1990) and on the horizontal one (Guerazzi, 1987; Nalesso, 1988; Johnscher-Fornasaro et al., 1990; Traldi \& Schlen7, 1990). On the other hand, the encrusting fauna on non-stabilized substrata is poorly known in Brazil, and there is no report on the species composition, abundance, vertical distribution or geographic variation. The only study on this kind of habitat was done by Rodrigues \& Shimizu (1988) and Shimizu \& Rodrigues (1988) who investigated the vagile fauna that uses the undersurface of boulders as a refuge against desiccation during low tides.

The main goal of this paper is to describe the encrusting fauna on boulder fields in two different shores, comparing the faunal distribution and diversity between the shores, tide levels and boulder sizes. Description of some new records of ascidian species from this habitat will be found elsewhere (Rocha \& Monniot, 1993, Rocha \& Monniot, in press).

\section{Study site}

The northern coast of São Paulo State has many sand beaches bordered by more or less extensive rocky shores. Boulders of various sizes are frequent beside rocky walls, from the intertidal region to the subtidal region .

This work was done on the northern coast of São Paulo State, at two shores situated in the São Sebastiao Channel (between $23^{\circ} 43^{\prime} \mathrm{S}$ and $23^{\circ} 53^{\prime} \mathrm{S}, 45^{\circ} 0^{\prime} \mathrm{W}$ and $45^{\circ} 27^{\prime} \mathrm{W}$ ): Praia Grande and Ponta do Baleeiro (Fig. 1).

Ponta do Baleeiro is located in a environmental protected area (Centro de Biologia Marinha - CEBIMar, USP), therefore less subjected to antropic interference. It is also less exposed to the waves than Praia Grande. The boulder field is $18 \mathrm{~m}$ long (parallel axis to the shore) and $7 \mathrm{~m}$ wide. The boulders are usually round or oval, with different levels of flatness. Among boulders we can find medium sized granules of sand.

Praia Grande is very appreciated by tourists, who easily reach the study site during low tides. The boulder field is approximately $10 \mathrm{~m}$ long in the high intertidal region to $20 \mathrm{~m}$ long in the subtidal region (parallel axis to the shore), and is $10 \mathrm{~m}$ wide. Boulders are either round, oval, or angulate with flattened faces. Among them we can find large size granules of sand.

No difference in boulders porosity was detected between the shores. The abrasion power of the sand was neither significantly different between shores.
Hydrodynamics (see methods) was greater at Praia Grande for six times in a total of twelve which were evaluated; and it was greater at Ponta do Baleeiro only once. There was no perceptible seasonal pattern. Great storms, longer than two days, were rare and occured in April, May, June and September in 1990, and between September and October in 1991.

Superficial water temperature, measured daily next to the laboratory at CEBIMar, varied from $20^{\circ} \mathrm{C}$ in the end of winter (September or October) to $26,5^{\circ} \mathrm{C}$ in fall (April or May) during the study period. Salinity kept values between 33 and $35 \%$.

\section{Materials and methods}

Characterization of environmental conditions was done according to the following parameters: water temperature and salinity, hydrodynamics, abrasion power of the sand and habitat heterogeneity. The boulder features studied were: undersurface area (small: $20-30 \mathrm{~cm}^{2}$ and large: $160-220 \mathrm{~cm}^{2}$ ), location: medium (MI) or low (LI) intertidal level, porosity and the intensity of disturbance caused by waves (displacement and overturning). Results on recruitment experiments are found in Rocha (1994), and those on disturbance, and transposition of boulders in Rocha (1993).

Hydrodynamics between the shores was compared using the difference of weight before and after the submersion of plaster half-spheres (6 cm diameter). Ten of them were placed in each shore, for 24 hours. The shores were compared with one-factor covariance analyses (ANCOVA), considering initial weight as the co-variable and the two shores as the levels of the treatment.

The boulders were found over other boulders, directly over sand, buried, or even immobilized by other boulders, creating at least four different microhabitat conditions for the undersurface community. To quantify this kind of environmental heterogeneity, the percentage of boulders in each of these four situations was calculated for every month. A standard deviation (SD) of the percentage values of 50 would correspond to low heterogeneity (all boulders in the same situation) and a SD of zero, to high heterogeneity (all boulders equally distributed among the four situations).

The community was monitored monthly during 1990 and 1991. Initially, ten boulders of each size, from each tide level, were permanently marked with commercial epoxi cement with a low relief number written on it. Due to the difficulty in locating all the marked boulders in each visit, more boulders were marked to increase the probability of finding at least eight. 


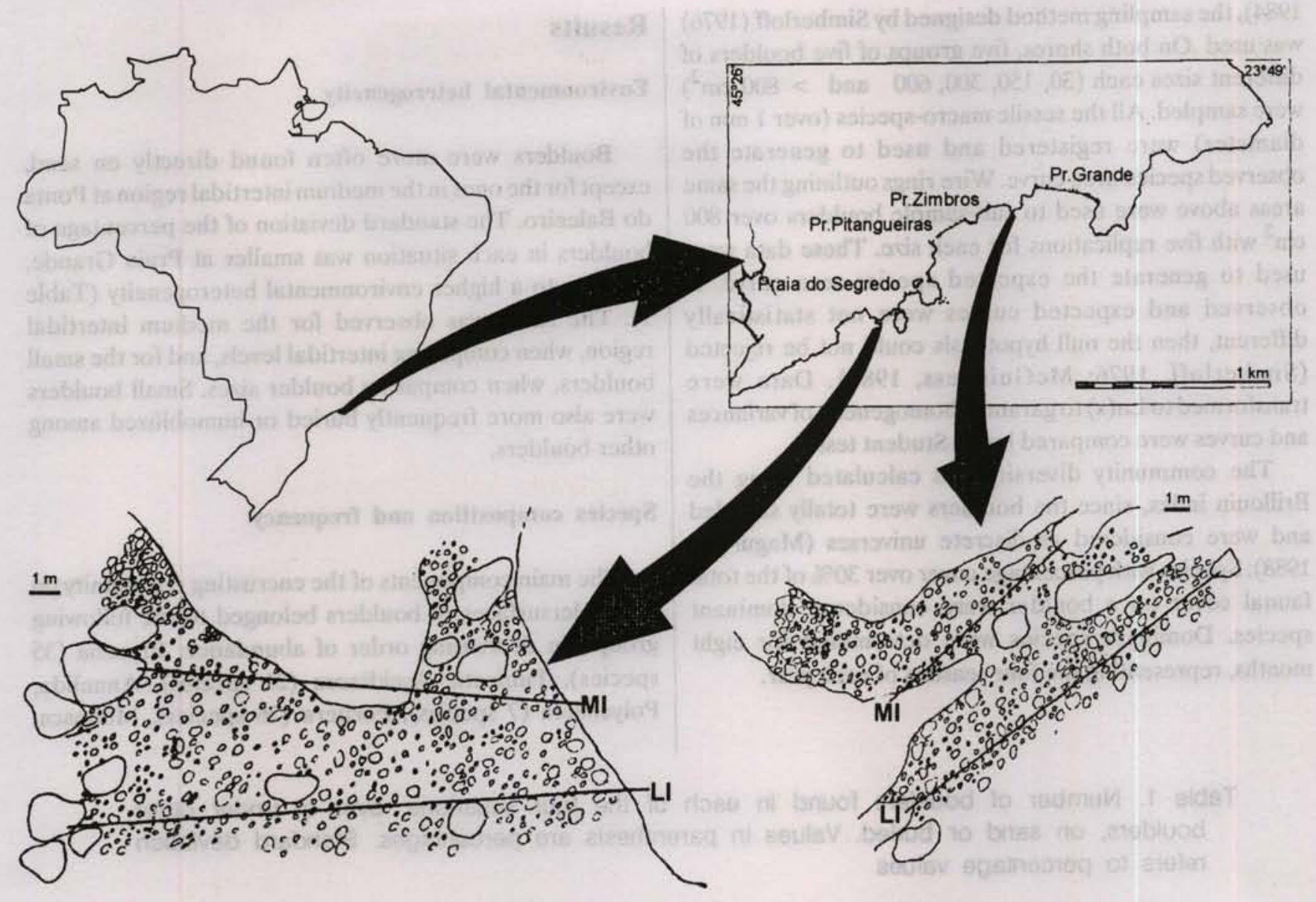

Fig. 1. Map of the region studied at São Sebastiano Channel with the detailed map of the boulder fields studied $(\mathrm{A}=$ Ponta do Baleeiro, $\mathrm{B}=$ Praia Grande, $\mathrm{MI}=$ medium intertidal zone, $\mathrm{LI}=$ low intertidal zone).

To observe the community, I placed each boulder upside down in a bowl filled with sea water. A quadrat with PVC frame and nylon mesh (holes of $1 \mathrm{~cm}^{2}$ ) was placed over the undersurface of the boulder and the area covered by each organism was measured counting the number of squares occupied. This method was used both for compound and solitary organisms since they were often fixed side by side (otherwise I calculated the cover surface as if they were fixed side by side). The percentage cover of the species was the result of the fraction between the number of squares occupied by each species by the total undersurface area $\left(\mathrm{cm}^{2}\right)$ of each boulder.

Some species were grouped together for the data analysis because of the difficulty to set them appart during field work. The Bryozoa Calloporidae and Membraniporidae were grouped and called "net Bryozoa", the species Stylopoma spongites, Schizoporella unicomis and Hipodiplosia americana were grouped as Schizoporellidae, all the arborescent bryozoa were also grouped together. Among ascidians, Didemnum speciosum and Lissoclinum fragile were grouped under
Didemnidae. Among sponges, species 13,15 and 16 from Suberetidae family were put together under species 16 ; Reniera sp, Amphimedon viridis, Callyspongia sp, Myxilla mucronata, Clathria sp and Chondrilla nucula were observed in the study site but did not enter the analysis because they were not found on the marked boulders. Among polychaetes, the two species of Spirorbis were considered together, and two species were not identified, called here serpulid 1 and 2 . Empty shells of bivalves were considered for the analysis of total percent cover of bivalves. Cnidarians species did not entered the analysis because of their little abundance and difficulty of identification during field work.

Since boulders are discrete substrates with limited area, it was possible to study the effect of available space on the number of species. To verify whether the number of species was a consequence of random sampling (null hypothesis), that is, smaller areas would receive fewer colonists and, therefore, fewer species (Connor \& McCoy, 1979; Coleman et al., 1982) or whether there were other factors influencing species-area curve (McGuinness, 
1984), the sampling method designed by Simberloff (1976) was used .On both shores, five groups of five boulders of different sizes each $\left(30,150,300,600\right.$ and $\left.>800 \mathrm{~cm}^{2}\right)$ were sampled. All the sessile macro-species (over $1 \mathrm{~mm}$ of diameter) were registered and used to generate the observed species-area curve. Wire rings outlining the same areas above were used to sub-sample boulders over 800 $\mathrm{cm}^{2}$ with five replications for each size. These data were used to generate the expected species-area curve. If observed and expected curves were not statistically different, then the null hypothesis could not be rejected (Simberloff, 1976; McGuinness, 1984). Data were transformed to $\mathrm{Ln}(\mathrm{x})$ to garantee homogeneity of variances and curves were compared by a t-Student test.

The community diversity was calculated using the Brillouin index, since the boulders were totally sampled and were considered as discrete universes (Magurran, 1988). Species with percentage cover over $30 \%$ of the total faunal cover on a boulder were considered dominant species. Dominant species were determined for eight months, representing the four seasons of each year.

\section{Results}

\section{Environmental heterogeneity}

Boulders were more often found directly on sand, except for the ones in the medium intertidal region at Ponta do Baleeiro. The standard deviation of the percentage of boulders in each situation was smaller at Praia Grande, pointing to a higher environmental heterogeneity (Table 1). The same was observed for the medium intertidal region, when comparing intertidal levels, and for the small boulders, when comparing boulder sizes. Small boulders were also more frequently buried or immobilized among other boulders.

\section{Species composition and frequency}

The main components of the encrusting community on the undersurfaces of boulders belonged to the following groups, in decreasing order of abundance: Bryozoa (35 species), Tunicata, Ascidiacea (21 species), Annelida, Polychaeta ( 7 species), Porifera (26 species), Mollusca,

Table 1. Number of boulders found in each of the four situations: over or under other boulders, on sand or buried. Values in parenthesis are percentages. Standard deviation refers to percentage values

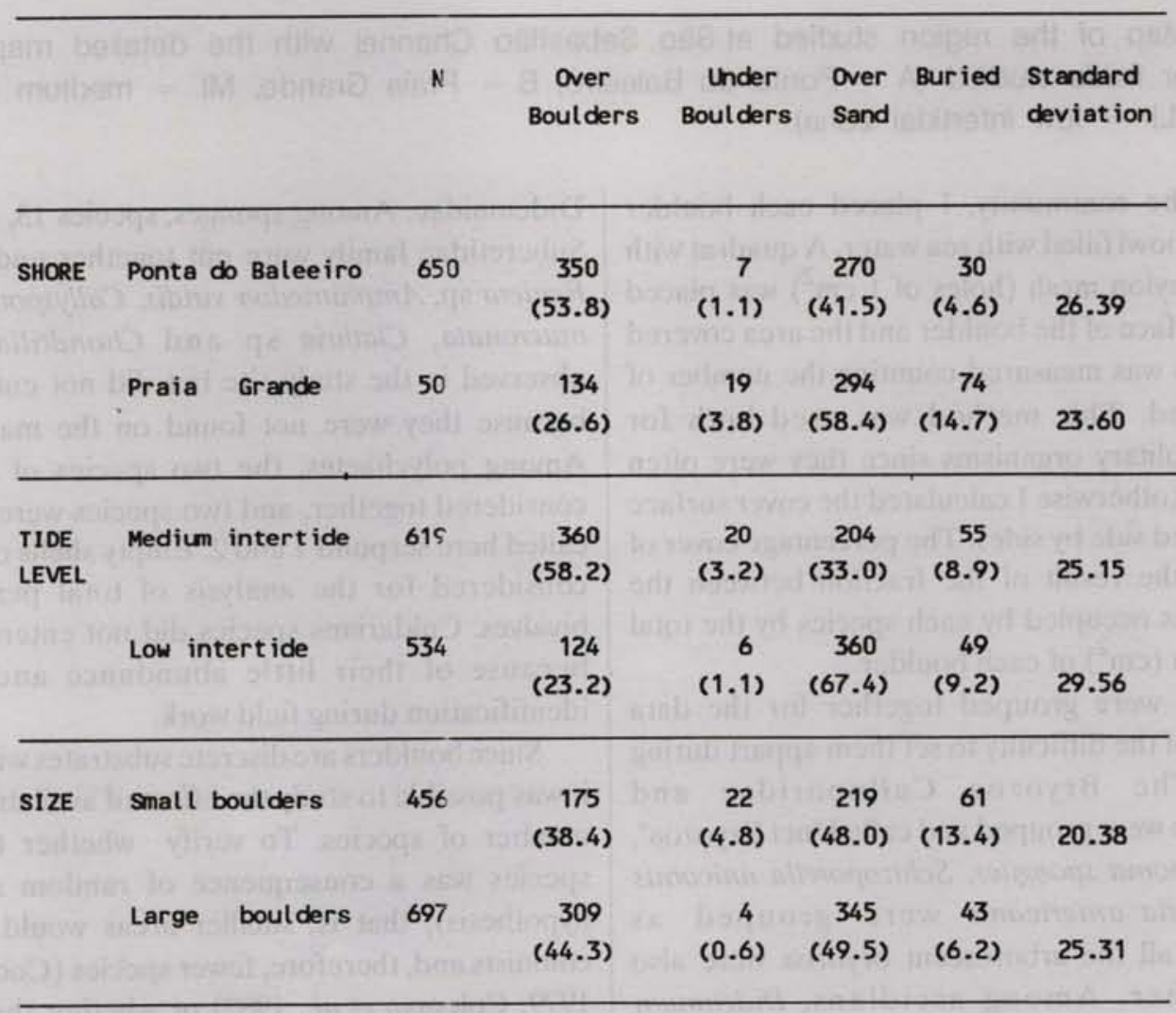


Bivalvia (2 species), Cnidaria, Hydrozoa (5 species), and Crustacea (Cirripedia and Amphipoda tubes not identified) (see Appendix I for a complete list of species).

Most species occured more frequently at Ponta do Baleeiro, in the low intertidal region and on large boulders (AppendixI). Few species occured exclusively in one of the shores (14 at Ponta do Baleeiro and 2 at Praia Grande) showing a similarity between their faunas. Quite more species occured exclusively in a tide level ( 37 species in the low intertidal region) or boulder size ( 25 species on large

\section{Appendix I}

boulders), indicating that those are more important features of the environment for the encrusting community.

\section{Main groups abundance}

The fauna had higher percentage cover values on large boulders in the low intertidal region, and minimal values in the medium intertidal region, specially for small boulders (Fig. 2). The flora had high percentage cover values on small boulders in the medium intertidal region. Empty substrate was abundant in all situations, mainly on small boulders and in the medium intertidal region.

BRYOZOA

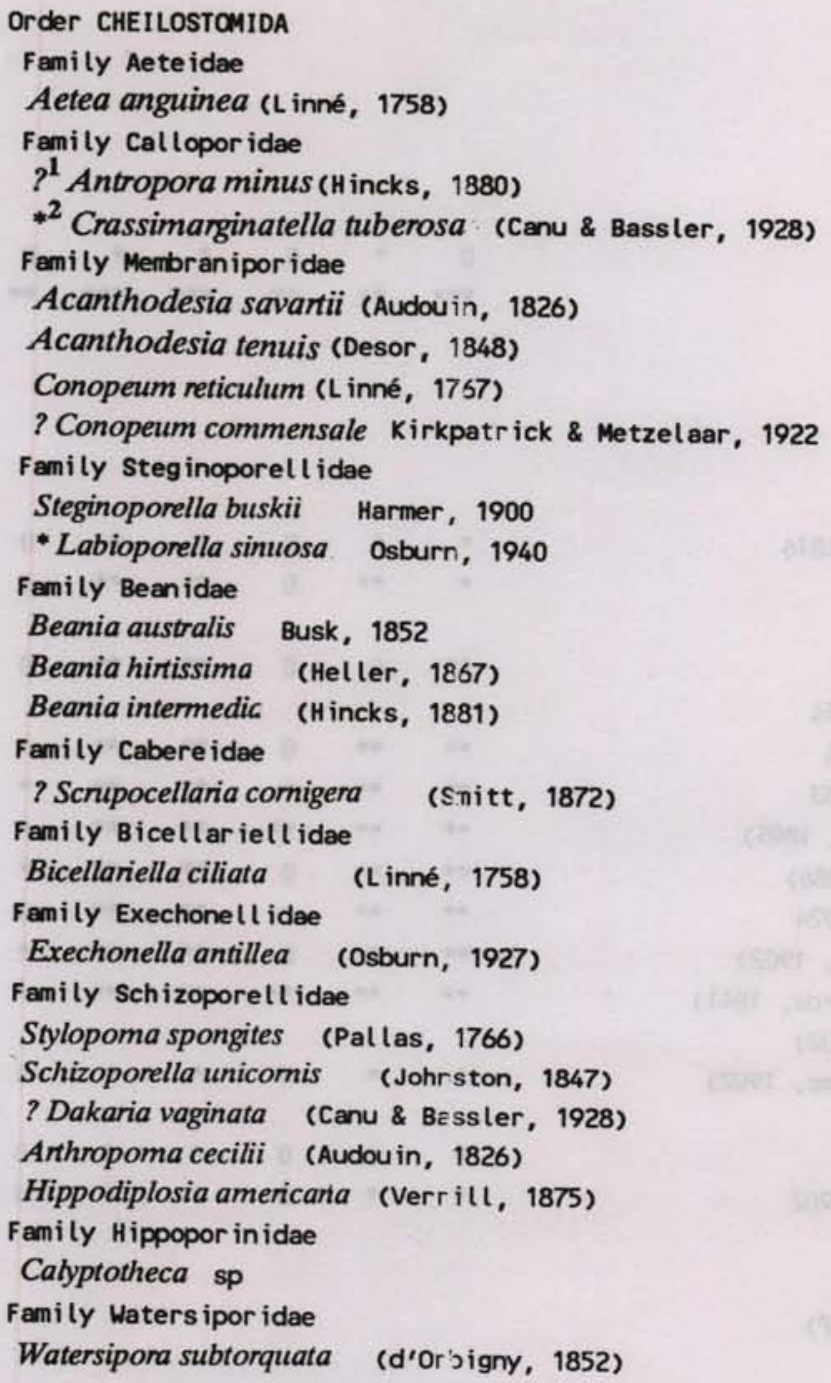


Appendix I - Cont.

Family Smitt inidae

Parasmittina trispinosa Johnston, 1838

Family Sertell idae

Cleidochasma porcellanum (Busk, 1860)

Family Catenicell idae

Catenicella contei (Audouin, 1826)

Family Savignyell idae

Savignyella lafontii (Audouir, 1826)

Family Celleporariidae

Celleporaria mordax (Marcus, 1937)

Celleporaria atlantica (Busk, 1884)

Family Phidolopor idae

Rhynchozoon verruculatum (Smitt, 1873)

Order CTENOSTOMIDA

Family Arachnidi idae

Nolella gigantea (Busk, 1856)

Family Vesicularridae

* Amathia vidovici (Heller, 1367)

Order CYCLOSTOMIDA

Family Crisi idae

Crisia ficulnea Buge, 1979

arborescent bryozoans

net bryozoans

BAL $^{3}$ PG MI LI $\quad$ L $\quad S$

ASCIDIACEA

Order APLOUSOBRANCHIA

Family Polycl inidae

Polyclinum constellatum Savicาy, 1816

Aplidium sp

Family Didem idae

* Didemnum ahu Monniot, 1987

- Didemnum granulatum Tokioke, 1954

* Didemnum lutarium Van Name, 1924

*Didemnum perlucidum Monniot, 1983

Didemnum psammathodes (sluiter, 1895)

Didemnum speciosum (Herdnan, 1886)

Didemnum vanderhorsti Van Nar a, 1924

Trididemnum orbiculatum (Van Name, 1902)

Diplosoma listerianum (Milne-Edwards, 1841)

Lissoclinum fragile (Van Name, 1902)

Polysyncraton amethysteum (Veา Name, 1902)

Family Polycitoridae

Clavelina oblonga Herdman, 1830

Distaplia bermudensis Van Narre, 1902

Order PHLEBOBRANCHIA

Family Cionidae

Ciona intestinalis

(L innaeus, 1767)

Family Ascidi idae

Phallusia nigra Savigny, $181 \epsilon$

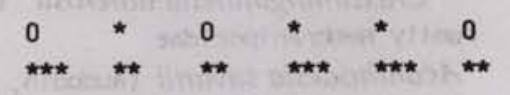


Appendix I - Cont.

Order STOLIDOBRANCHIA

Family Styel idae

Botryllus niger (Hercman, 18\&5)

Botryllus giganteus Aron \& Scle Cava, 1991

Botryllus tabori Rodr igues, 1962

Eusynstyela tincta (Van Name, 1902)

Symplegma brakenhielmi (Micheelsen, 1904)

BAL $^{3}$ PG MI LI L $\quad S$

PORIFERA

\section{Order DICTYOCERATIDA}

Family Spongi idae

species 1 (rose)

species 2 (half-sphere, be ige)

Order HAPLOSCLERIDA

Family Haliclonidae

Adocia carbonara Lamarck, 1814

Adocia sp (l inear, be ige)

Reniera sp

Haliclona group: species 4 (crust, beige)

species 5 (brown or dark grey)

species 6 (thick, yellow)

Family Niphat idae

Amphimedon viridis Duchassairg \& Michelotti, 1864

Family Callyspongi idae

Callyspongia sp

Order POECILOSCLERIDA

Family Mycalidae

Mycale (Aegogropila) americana Van Soest, 1984

Mycale (Carmia) microsigmatos 1 Arndt, 1927

species 10 (brown or violet)

Family Myxill idae

Myxilla sp

Myxilla mucronata Pul itzer-F inal i, 1986

Family $\mathrm{Clathri}$ idae

Clathria sp

aff. Fam. Desmacellidae

species 11 (thin and smooth crust, red)

aff. Fam. Microcionidae

species 12 (brownish red)

Family Ophl itaspong i idae

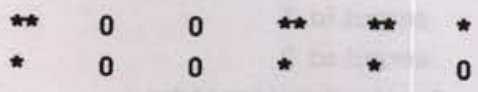

Artemisina aff. melana

Order HADROMERIDA

Family Suberitidae

species 13 (yellow, oscules and channels well defined)

species 14 (smooth black crust)

species 15 (thin smooth oranç crust)

species 16 (very thin and smc sth crust, yellow or orange) * Order CHONDROSIDA

Family Chondros i idae

Chondrilla nucula Schmidt, 1870

Classe CALCAREA 
Appendix I - Cont.

Order SYCETIIDA

Family Sycettidae

? Sycon sp

POLYCHAETA

Família Serpul idae

Spirorbis moerchi Levinsen, 1983

Spirorbis (Paralaeospira) calypso) Z Zibrowius, 1969

Spirorbiss spp

Hydroides brachyacantha. Rio je, 1941

Pomatoceros minutos Rioja, 1942

serpul id 1

serpul id 2

Family Sabellariidae

Phragmatopoma lapidosa Kinberg, 1867

Family Terebell idae

Polycirnus sp

Family Eunicidae

Eunice tubifex Crossland, 19C4

Family Cirratul idae

Family Sabell idae

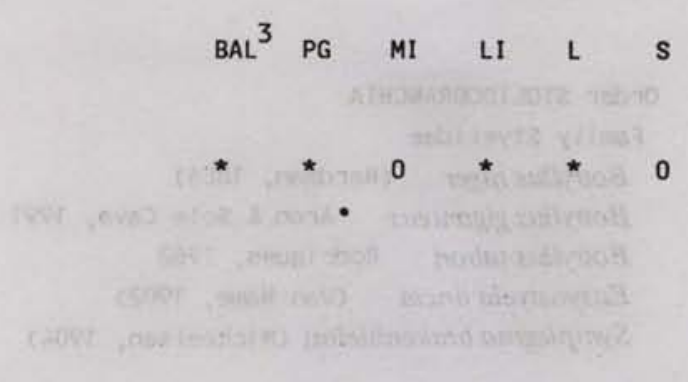

MOLLUSCA

Classe Bivalvia

Family Ostreidae

Ostrea puelchana Orb., 1841

Family Chamidae

Chama congregata Conrad, 183?

CNIDARIA

Classe Hydrozoa

Subclasse Athecatae

Family Eudendri idae

Eudendrium sp

Subclasse Thecatae

Family Halopter i idae

Halopteris diaphana (Heller, 1868)

Family Kirchenpauer $\mathrm{i}$ idae

Kirchenpaueria halecioides ( $f$ Lder, 1859)

Family Campanular i idae

Clytia sp

Obelia dichotoma (L innaeus, 1758)

$T_{?}=$ uncertain identification

$2 *$ new report for Brazil

3 Frequency is relative to all boulders observed during the study period: $0=$ absence of the species, $*=$ less then $1 \%, \star \star=1-10 \%, \star \star \star=10-20 \%, \star \star \star \star=$ more than $20 \%$ of boulders with the species. $B A L=$ Ponta do Bale ziro, $P G=$ Praia Grande, MI = medium intertidal zone, LI = loi intertidal zone, $L=$ large boul jers, $S=$ small boulders .

Species without frequency indi ated were grouped with other species, or could not be identified in the field, or were present in the study site but not on marked boulders (see text). 

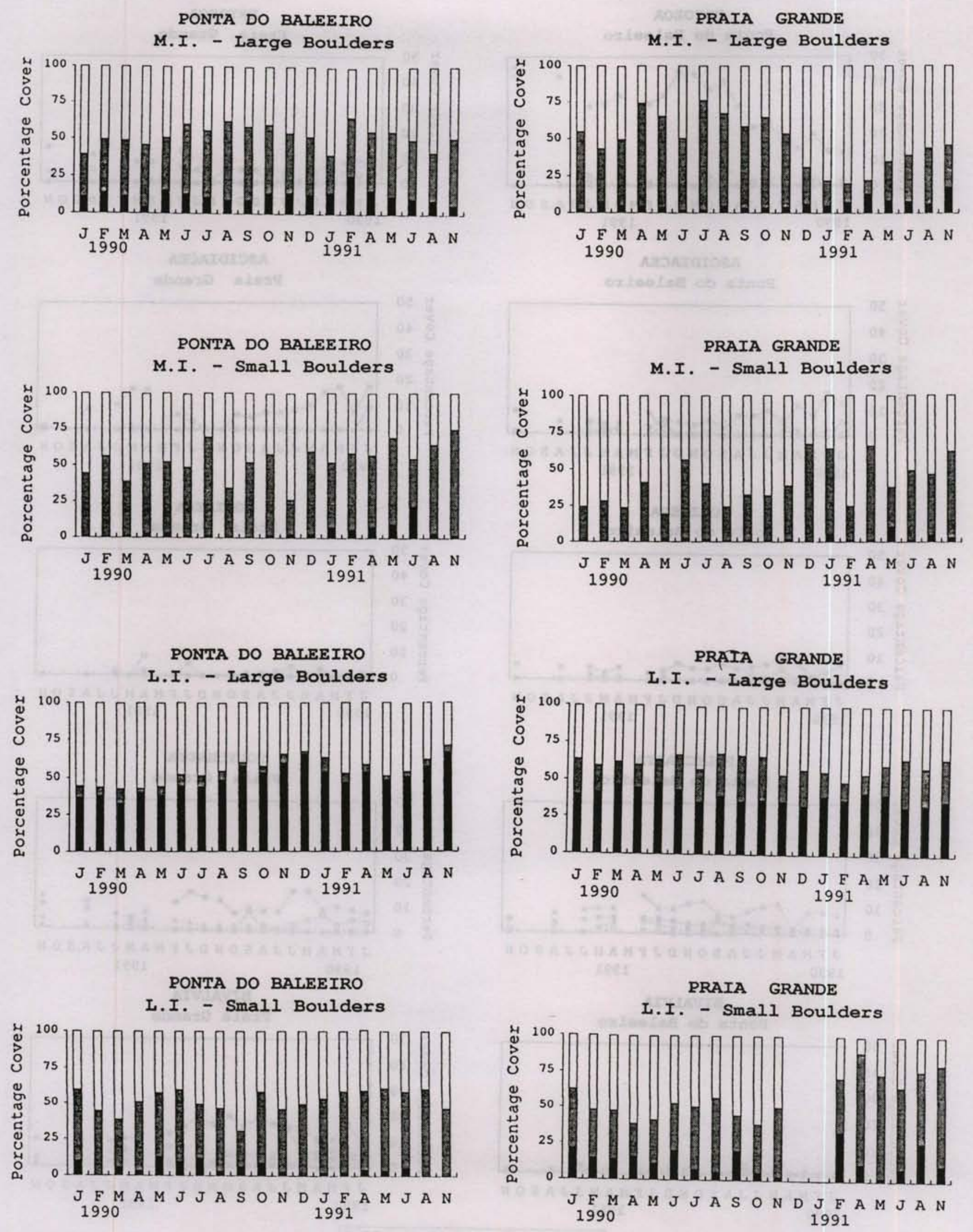

- Fauna EFlora cBare space

Fig. 2. Percentage cover of total fauna, flora and empty space on boulders. 


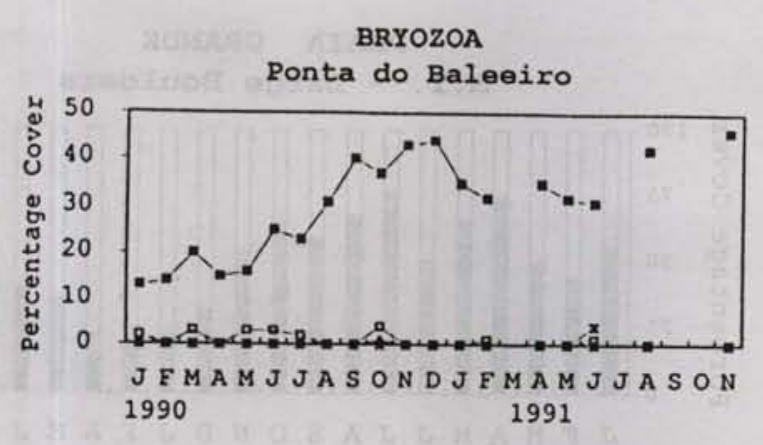

ASCIDIACEA

Ponta do Baleeiro

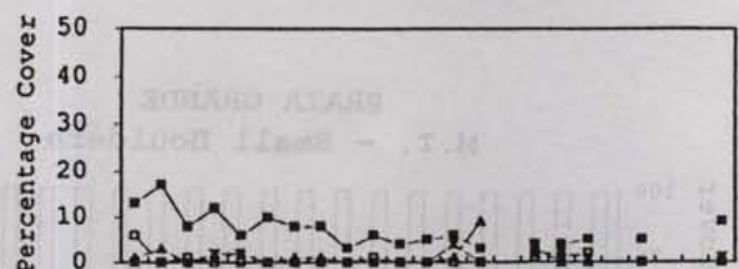

JFMAM J JASOND J FMAM J JASON 1990 1991

PORIFERA

Ponta do Baleeiro

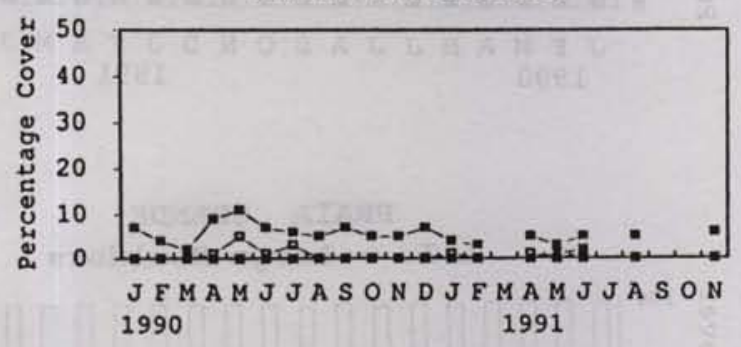

POLYCHAETA

Ponta do Baleeiro

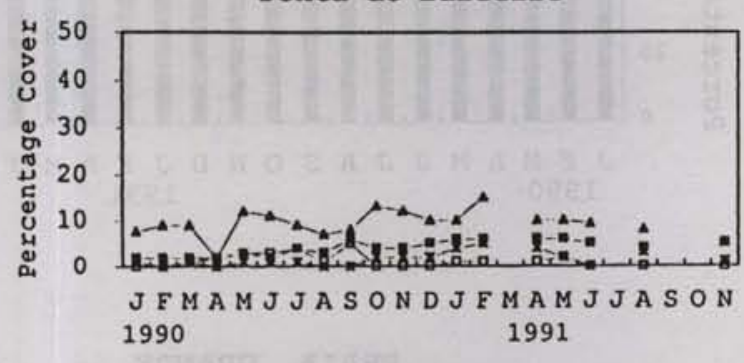

BIVALVIA

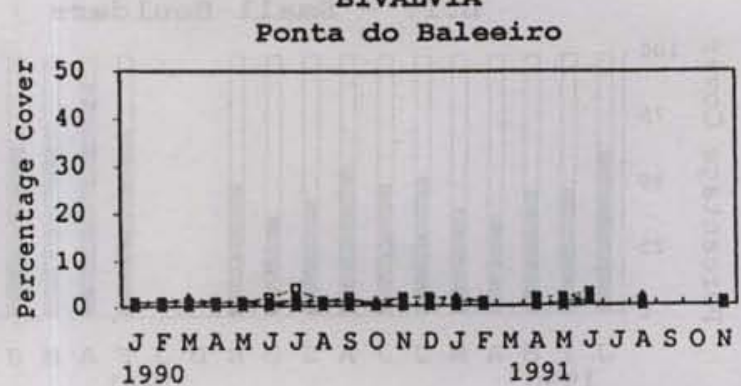

BRYOZOA

Praia Grande

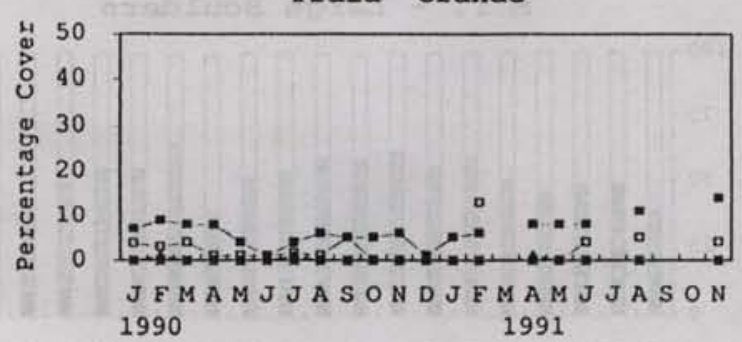

ASCIDIACEA

Praia Grande

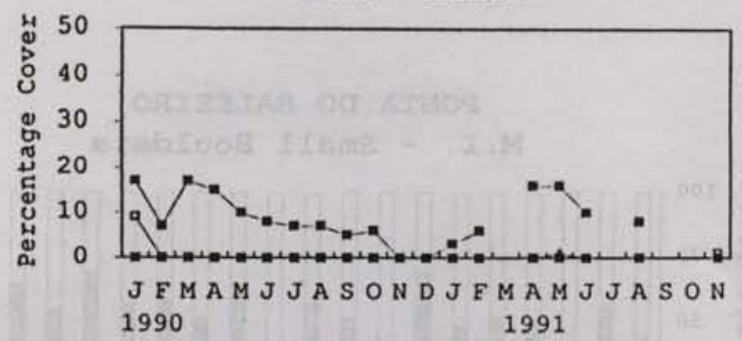

PORIFERA

Praia Grande

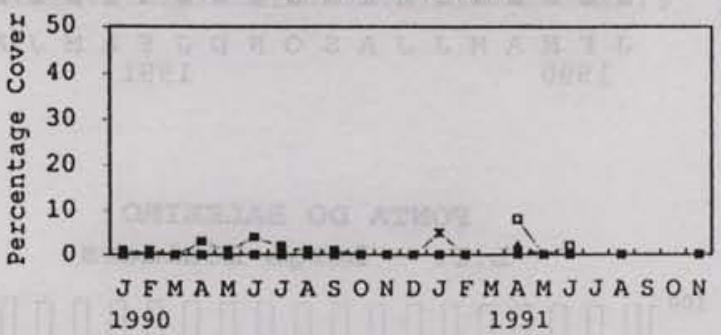

POLYCHAETA

Praia Grande

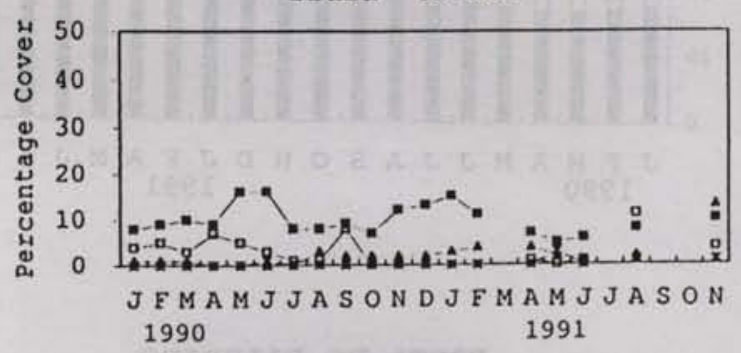

BIVALVIA

Praia Grande

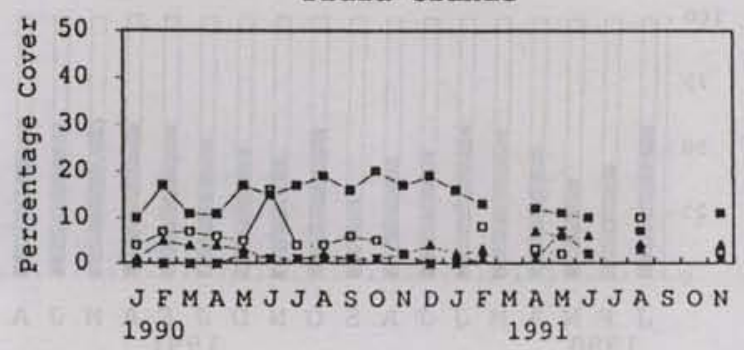

$-4-M I, L-\pi-M I, S-\infty-L I, L-0-L, I, S$

Fig. 3. Percentage cover of he five groups studied on each site (Ponta do Baleeiro or Praia Grande), tide level $(\mathrm{MI}=$ medium ir tertidal zone, $\mathrm{LI}=$ low intertidal zone) and boulders size $(\mathrm{S}=$ small, $\mathrm{L}=$ large). 
Bryozoans had low percentage cover in the medium intertidal region in both shores (Fig. 3). In the low intertidal region, cover was higher on large boulders of Ponta do Baleciro. The same pattern was observed for ascidians, but this group was a little more abundant at Praia Grande. Sponges had a significant cover only on large boulders in the low intertidal region at Ponta do Baleciro. Cnidarians' percentage cover was very small in all situations, with a single peak of abundance in May, 1991. Polychaetes had the same pattern described before at Praia Grande, with high percentage cover values on large boulders in the low intertidal region. However, at Ponta do Baleeiro, higher percentage cover values occured on the large boulders from the medium intertidal region. Bivalves were rare at Ponta do Baleeiro; at Praia Grande the highest values ohserved were again on large boulders in the low intertidal region.
There were variations in percintage cover along the year but the pattern did not correspond to the water temperature curve of the same period neither for the total fauna nor for any single group.

\section{Species-area curve}

On both shores there was a positive relationship between species number and area, both for boulders and for sub-samples (Fig. 4). At Ponta do Baleiro, regression curves of boulders and sub- samples were not statistically different ( $t$-Student $=-0.268, p=0.79$ ). At Praia Grande, however, the number of species on boulders was greater than from sub-samples ( $\mathrm{t}$-Student $=2.053, \mathrm{p}=0.047$ ).

\section{Diversity and dominance}

In general, community diversity at Ponta do Baleeiro was greater than at Praia Grande, the higher deviations
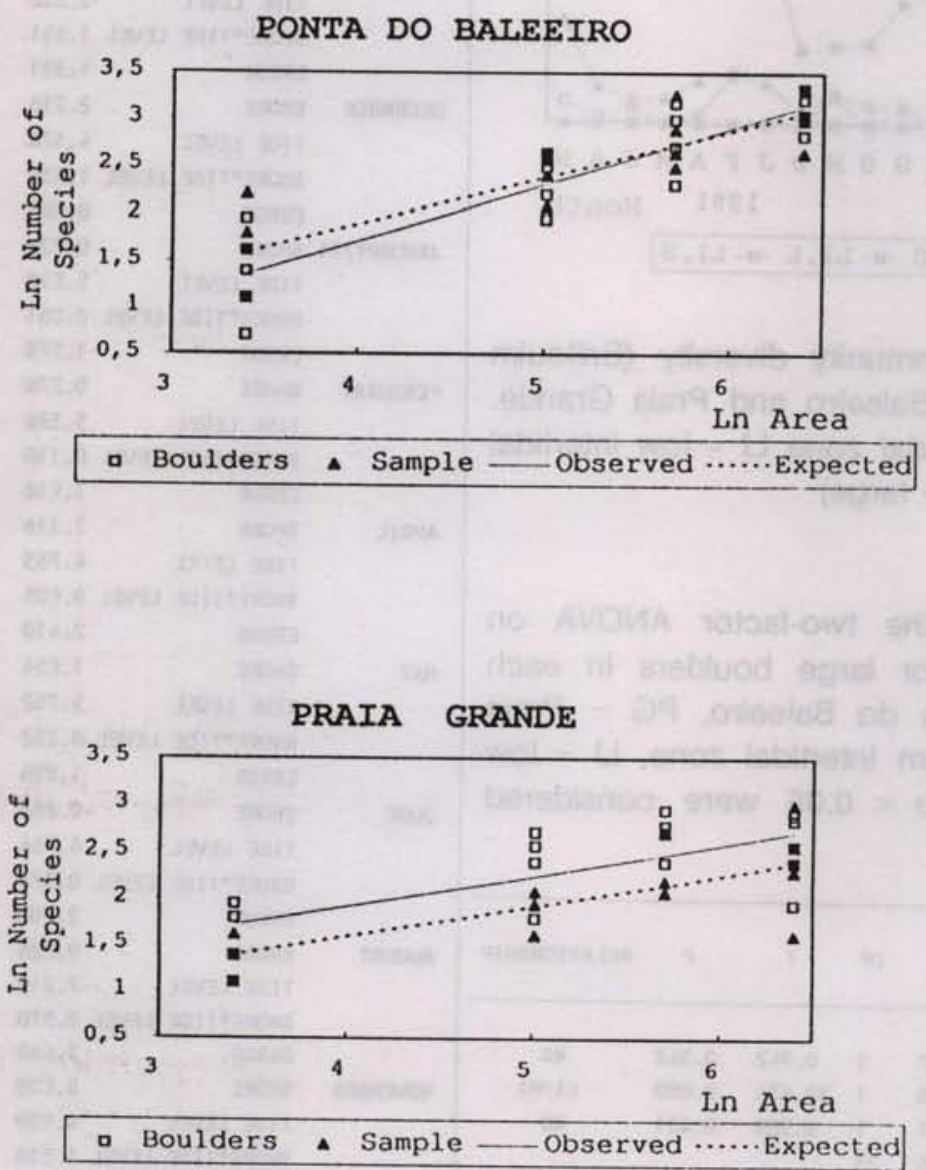

Fig. 4. Variation of the number of species on boyh boulders (observed) and sub-samples of large boulders (expected). with the undersurface area 
seen among large boulders in the low intertidal region (Fig. 5). Small boulders had low community diversity in all situations. Variation in diversity showed neither any seasonal pattern, nor correspondence to the temperature curve. Diversity on large boulders was compared through a two-factor ANOVA for each month (Tab. 2). In
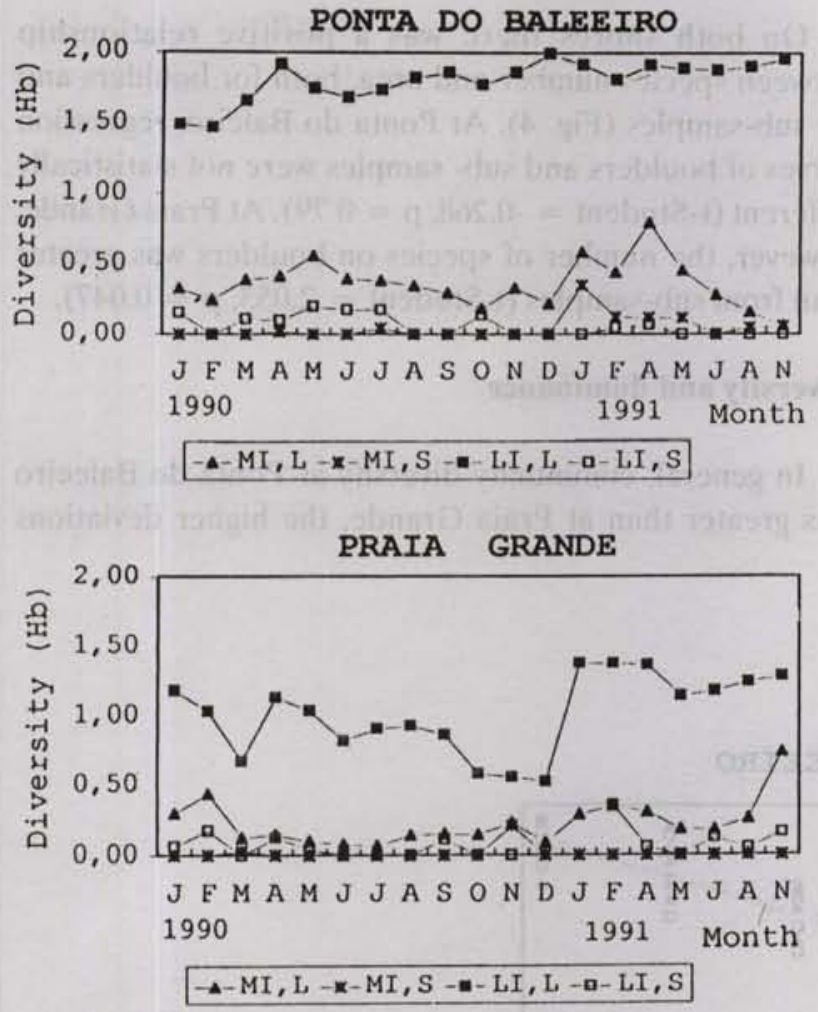

Fig. 5. Encrusting community diversity (Brillouim index) on Ponta do Baleeiro and Praia Grande. (MI = Medium intertidal zone, $\mathrm{LI}=$ low intertidal zone, $S=$ small, $\mathrm{L}=$ large)

Table 2. Results of the two-factor ANOVA on comunity diversity for large boulders in each month. $\mathrm{BAL}=$ Ponta do Baleeiro, $\mathrm{PG}=$ Praia Grande; $\mathrm{MI}=$ medium intertidal zone, $\mathrm{LI}=$ low intertidal zone (all $\mathrm{p}<0,05$ were considered significant)

\begin{tabular}{|c|c|c|c|c|c|c|}
\hline & SOURCES & sa & DF & $\mathbf{F}$ & $\mathbf{P}$ & RELATIONSHIP \\
\hline \multirow[t]{4}{*}{ JANUARY $/ 90$} & SHORE & 0.177 & 1 & 0.942 & 0.342 & NS \\
\hline & TIDE LEVEL & 6.138 & 1 & 32.634 & 0.000 & $L I>M I$ \\
\hline & SHORE*TIDE LEVEL & 0.111 & 1 & 0.589 & 0.451 & NS \\
\hline & ERROR & 4.326 & 23 & & & \\
\hline \multirow[t]{4}{*}{ FEBRUARY } & SHORE & 0.133 & 1 & 0.640 & 0.430 & NS \\
\hline & TIDE LEVEL & 6.596 & 1 & 33.660 & 0.000 & $L I>M I$ \\
\hline & SHORE*TIDE LEVEL & 0.824 & 1 & 3.962 & 0.055 & NS \\
\hline & ERROR & 6.443 & 31 & & & \\
\hline
\end{tabular}

Table 2. Cont.

\begin{tabular}{|c|c|c|c|c|c|c|c|}
\hline & SOURCES & & sa & DF & $\mathbf{F}$ & $\mathbf{P}$ & RELATIONSHIP \\
\hline \multirow[t]{4}{*}{ MARCH } & SHORE & & 3.314 & 1 & 17.834 & 0.000 & $B A L>P G$ \\
\hline & TIDE LEVEL & & 6.507 & 1 & 37.164 & 0.000 & $\mathrm{LI}>\mathrm{MI}$ \\
\hline & SHORE*TIDE & LEVEL & 1.101 & 1 & 5.926 & 0.021 & \\
\hline & ERROR & & 5.576 & 30 & & & \\
\hline \multirow{4}{*}{ APRIL } & SHORE & & 1.939 & 1 & 15.065 & 0.001 & $B A L>P G$ \\
\hline & TIDE LEVEL & & 10.732 & 1 & 83.376 & 0.000 & LI $>M I$ \\
\hline & SHORE*TIDE & LEVEL & 0.469 & 1 & 3.647 & 0.068 & NS \\
\hline & ERROR & & 3.089 & 24 & & & \\
\hline \multirow[t]{4}{*}{ MAY } & SHORE & & 2.598 & 1 & 12.539 & 0.001 & $B A L>P G$ \\
\hline & TIDE LEVEL. & & 8.463 . & 1 & 40.847 & 0.000 & $L I>M I$ \\
\hline & SHORE^TIDE & LEVEL & $0.120^{\circ}$ & 1 & 0.581 & 0.452 & NS \\
\hline & ERROR & & 5.574 & 27 & & & \\
\hline \multirow[t]{4}{*}{ JUNE } & SHORE & & 2.407 & 1 & 11.952 & 0.002 & $B A L>P G$ \\
\hline & TIDE LEVEL & & 6.599 & 1 & 34.746 & 0.000 & $\mathrm{LI}>\mathrm{MI}$ \\
\hline & SHORE*TIDE & LEVEL & 0.514 & 1 & 2.551 & 0.123 & NS \\
\hline & ERROR & & 4.834 & 24 & & 117 & 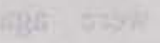 \\
\hline JULY & ............ & & & & & & 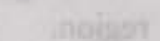 \\
\hline AUGUST & (.......... & & & & & & 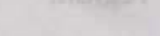 \\
\hline SEPTEMBER & 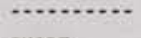 & & & & & & \\
\hline \multirow[t]{4}{*}{ OCTOBER } & SHORE & & 3.554 & 1 & 26.291 & 0.000 & $B A L>P G$ \\
\hline & TIDE LEVEL & & 9.520 & 1 & 70.426 & 0.000 & $L I>M I$ \\
\hline & SHORE*TIDE & LEVEL & 3.117 & 1 & 23.061 & 0.000 & \\
\hline & ERROR & & 4.461 & 33 & & & \\
\hline \multirow[t]{4}{*}{ NOVEMBER } & SHORE & & 2.111 & 1 & 19.868 & 0.000 & $B A L>P G$ \\
\hline & TIDE LEVEL & & 3.652 & 1 & 34.365 & 0.000 & LI>MI \\
\hline & SHORE*TIDE & LEVEL & 1.551 & 1 & 14.595 & 0.002 & \\
\hline & ERROR & & 1.381 & 13 & & & \\
\hline \multirow[t]{4}{*}{ DECEMBER } & SHORE & & 2.216 & 1 & 23.006 & 0.001 & $B A L>P G$ \\
\hline & TIDE LEVEL & & 4.176 & 1 & 43.357 & 0.000 & $L 1>M I$ \\
\hline & SHORE*TIDE & LEVEL & 1.555 & 1 & 16.138 & 0.002 & \\
\hline & ERROR & & 0.963 & 10 & & & \\
\hline \multirow[t]{4}{*}{ JANUARY/91 } & SHORE & & 0.726 & 1 & 5.521 & 0.037 & $B A L>P G$ \\
\hline & TIDE LEVEL & & $5 . \varepsilon 58$ & 1 & 44.535 & 0.000 & $\mathrm{LI}>\mathrm{MI}$ \\
\hline & SHORE*TIDE & LEVEL & 0.061 & 1 & 0.462 & 0.510 & NS \\
\hline & ERROR & & 1.578 & 12 & & & \\
\hline \multirow[t]{2}{*}{ FEBRUARY } & SHORE & 6 & 0.276 & 1 & 1.585 & 0.234 & NS \\
\hline & TIDE LEVEL. & & 5.326 & 1 & 30.553 & 0.000 & LI>MI \\
\hline \multirow[t]{2}{*}{$x^{2}-x^{2}=1$} & SHORE*TIDE & LEVEL & 0.110 & 1 & 0.633 & 0.443 & NS \\
\hline & ERROR & & 1.518 & 11 & & & \\
\hline \multirow[t]{4}{*}{ APRIL } & SHORE & & 1.116 & 1 & 5.556 & 0.036 & $B A L>P G$ \\
\hline & TIDE LEIEL & & 4.753 & 1 & 23.714 & 0.000 & $\mathrm{LI}>\mathrm{MI}$ \\
\hline & SHORE*TIDE & LEVEL & $0 . \cos$ & 1 & 0.024 & 0.880 & NS \\
\hline & ERROR & & 2.410 & 12 & & & \\
\hline \multirow[t]{4}{*}{ MAY } & SHORE & & 1.054 & 1 & 6.772 & 0.023 & $B A L>P G$ \\
\hline & TIDE LEVEL & & 5.752 & 1 & 36.603 & 0.000 & $L I>M I$ \\
\hline & SHORE*TIDE & LEVEL & 0.232 & 1 & 1.475 & 0.248 & \\
\hline & ERROR & & 1.886 & 12 & & & \\
\hline \multirow[t]{4}{*}{ JUNE } & SHORE & & 0.662 & 1 & 3.598 & 0.082 & NS \\
\hline & TIDE LEVEL & & 6.764 & 1 & 36.760 & 0.000 & $\mathrm{LI}>\mathrm{MI}$ \\
\hline & SHORE*TIDE & LEVEL & 0.376 & 1 & 2.044 & 0.178 & \\
\hline & ERROR & & 2.208 & 12 & & & \\
\hline \multirow[t]{4}{*}{ AUGUST } & SHORE & & 0.324 & 1 & 2.477 & 0.141 & NS \\
\hline & TIDE LEVEL & 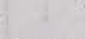 & 7.219 & 1 & 55.216 & 0.000 & $L I>M I$ \\
\hline & SHORE*TIDE & LEVEL & 0.570 & 1 & 4.358 & 0.059 & \\
\hline & ERROR & & 1.569 & 12 & & & \\
\hline \multirow[t]{4}{*}{ NOVEMBER } & SHORE & & 0.000 & 1 & 0.005 & 0.947 & NS \\
\hline & TIDE LEVEL & & 4.589 & 1 & 73.424 & 0.000 & $L I>M I$ \\
\hline & SHORE*TIDE & LEVEL & 1.530 & 1 & 22.521 & 0.001 & \\
\hline & ERROR & & 0.747 & 11 & & & \\
\hline
\end{tabular}


ten months, Ponta do Baleeiro was more diverse than Praia Grande, and in all months, the low intertidal region was more diverse than the medium intertidal region.

Community diversity on large boulders increased with percentage cover of the fauna, especially in the low intertidal region, for both shores (Fig. 6).

Besides the low percentage cover, small boulders were also rarely dominated by one species (Table 3 ). Large boulders in the medium intertidal region were usually dominated by polychaetes and bivalves (dead shells mostly) in both shores. In the low intertidal region, these boulders were dominated by bryoz.oans at Ponta do Baleeiro and bryozoans and bivalves (dead shells) at Praia Grande. Less frequently, ascidians, sponges and polychaetes also dominated boulders in the low intertidal region.

\section{Discussion}

The species composition of the encrusting community was similar in both shores, with differences in abundance for the various groups. These differences, however, were not greater than the variation between intertidal region levels or boulder sizes, indicating that these last factors are more influential on the species distribution.

Among the features studied, no differences were detected in porosity of the boulders nor in the abrasion capacity of the sand between the shores. Hydrodynamics and environmental heterogeneity were higher at Praia Grande.

The kind of environmental heterogeneity evaluated here was not responsible for creating diversity. Small boulders, the medium intertidal region and Praia Grande
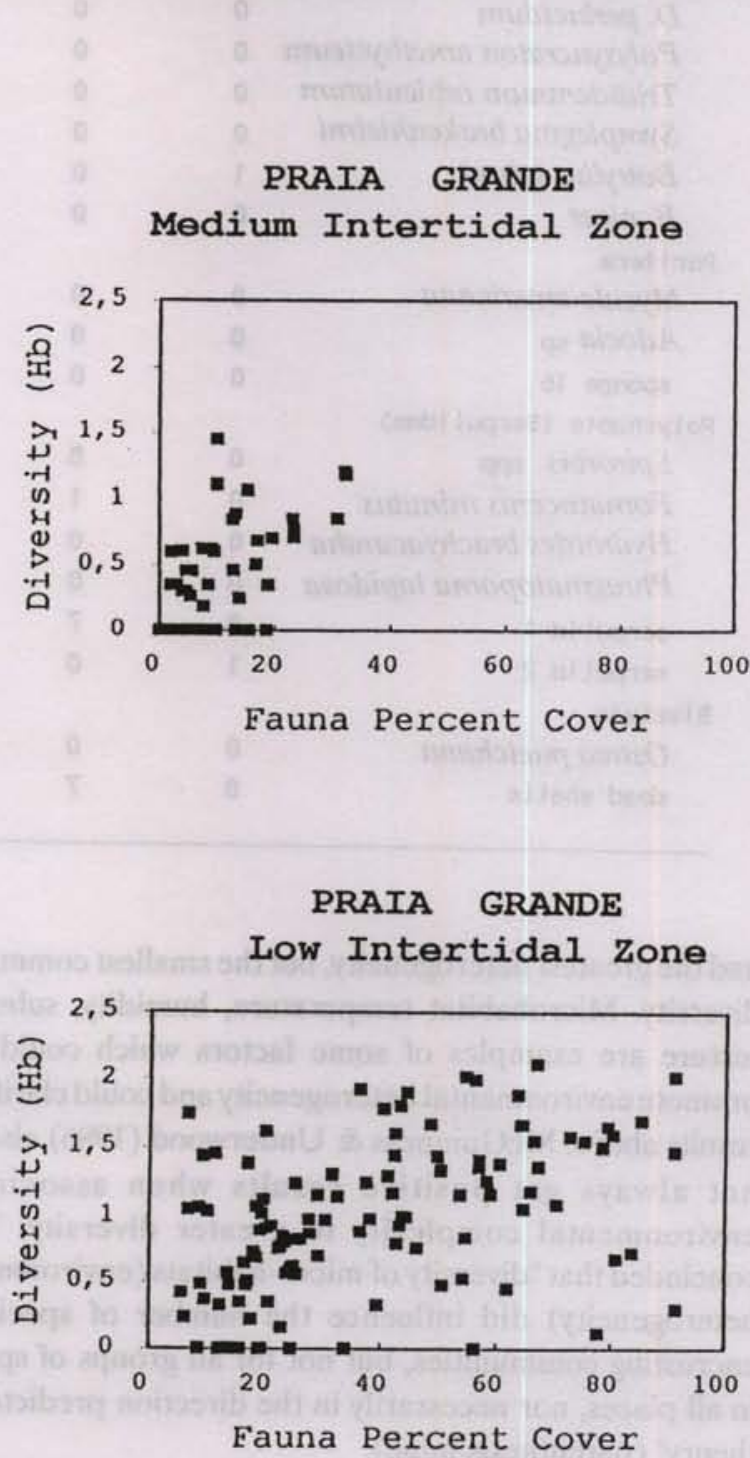

Fig. 6. Relationship between diversity (Brillouin index) and percentage cover of the fauna on large boulders. 
Table 3. Dominant species (see text for definition). Cells indicate the number of months (maximum of 8) each species appeared as dominant on at least one boulders

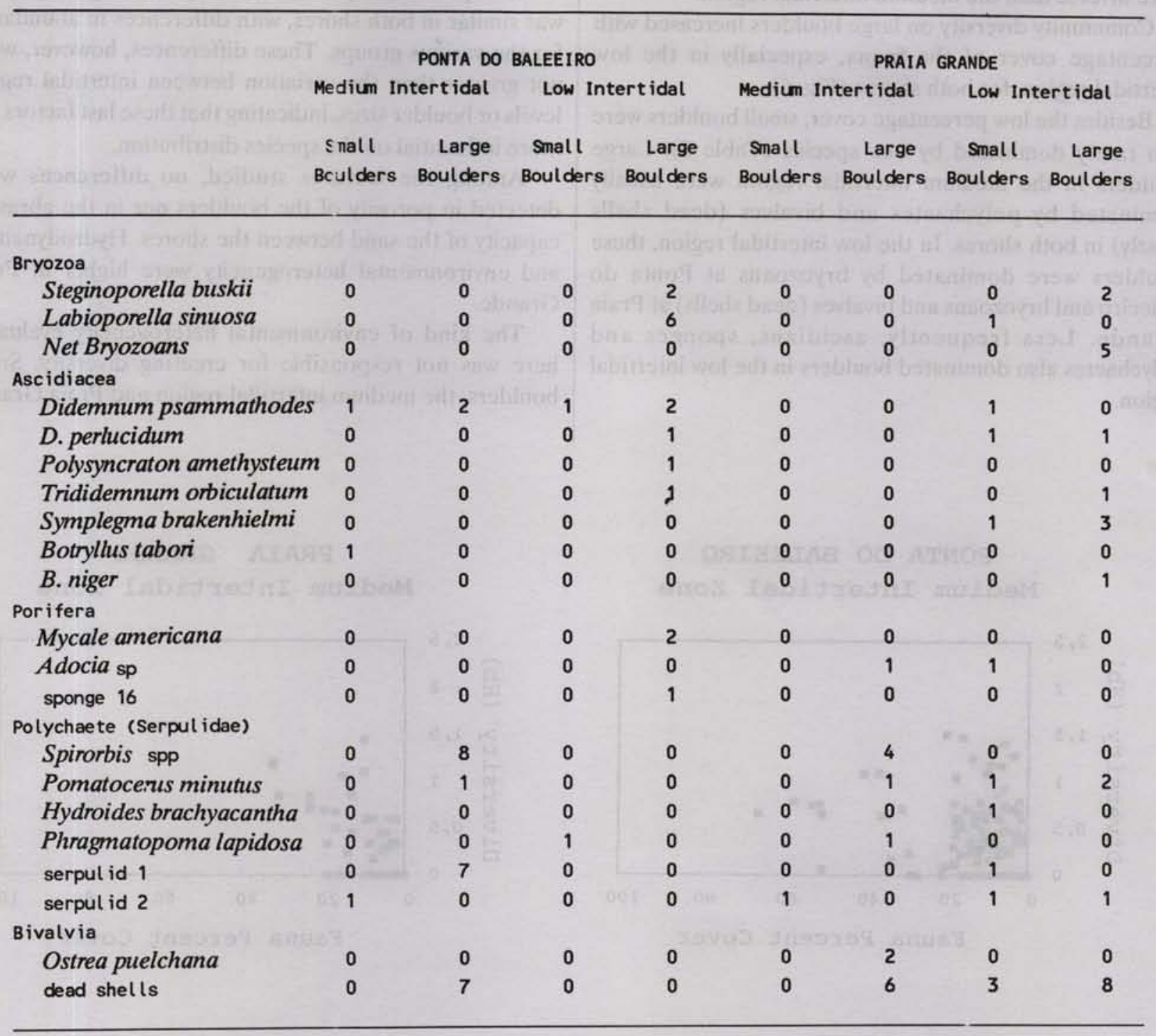

had the greatest heterogeneity, but the smallest community diversity. Microhabitat temperature, humidity, substrate texture are examples of some factors which could also promote environmental heterogencity and could clarify the results above. McGuinness \& Underwood (1986) also did not always get positive results when associating environmental complexity to greater diversity. They concluded that "diversity of micro-habitats (environmental heterogeneity) did influence the number of species in encrusting communities, but not for all groups of species in all places, nor necessarily in the direction predicted by theory" (parenthesis mine).

In general, the boulders had low percentage cover of fauna, and higher values were found on large boulders in the low intertidal region at Ponta do Baleeiro (32 to 65\% of space covered). Osman (1977) also found monthly means of percentage cover between 30 and $70 \%$, and McGuinness (1984, in McGuinness \& Underwood, 1986) only found almost $100 \%$ of cover on the undersurfaces of large boulders low on the shore. Some empty space was always present on boulders, indicating low importance of pre-emption of space or overgrowth competition for this community. Further, if competition was an intensive process, diversity should decrease with increasing percentage cover because good competitive species would dominate the space (Keen \& Neill, 1,ö). However, I observed the opposite situation, that is, diversity increased with increasing cover. Available space for colonization was also observed by Keen \& Neill (1980) on climax concrete blocks, and they speculated that this was due to seasonal shortages of primary space because of the heavy occupation during summer; the space would be vacated 
during the other seasons. It seems that it is not the case at São Sebastiāo, where space was available on the undersurfaces of boulders all year long.

The size of the boulder had a great influence on the distribution of species. Fouling community studies showed that the greater the size of the substrate, the greater the number of accumulated species (Schoener, 1974; Jackson, 1977; Osman, 1977, 1982). McGuinness (1984) discuss that 4 hypothesis should be considered to explain the increment of species number with area. They are: Passive Sampling or Random Placement Hypothesis (Connor \& McCoy, 1979; Coleman et al., 1982), the Equilibrium Theory of Island Biogeography (MacArthur \& Wilson, 1963, 1967), Habitat Diversity Hypothesis (Williams, 1964, in McGuinness, 1984), and the Intermediate Disturbance Hypothesis (Connell, 1978).

For the shores studied, there were two different patterns for the relation species number/boulder size. At Ponta do Baleeiro the mean number of observed species on boulders was similar to the expected number, so that the null hypothesis, that the number of species represent a sample of greater areas taken by chance, cannot be rejected. We should then conclude that no physical or biological processes is acting to create the pattern.

On the other hand, at Praia Grande the number of species observed on boulders was greater than the number expected, indicating the interference of other processes. According to McGuinness (1984), an observed curve over the expected curve indicates greater number of species on boulders of intermediate sizes, what is consistent with the Intermediate Disturbance Hypothesis (Connell, 1978). The comparison of hydrodynamics showed that Praia Grande is more exposed to water movement and, therefore, to disturbance of the boulders. Likewise, data from disturbance measurements corroborate that the boulders at Praia Grande are more subjected to displacement and overturning (to be reported elsewhere).

Boulder size can also influence dominance. Osman (1977) states that decreased substrate size increases the probability of short-term seasonal dominance of species with a seasonal peak of high growth rate, or of colonial species able to occupy a major proportion of the available space on small substrates at a much smaller size. However, Kay \& Keough (1981) and Keough (1984) observed a negative correlation between colonization and competition capabilities for the great taxonomic groups, in South Australia. They found that small substrates were easily found by better colonizers (bryozoans and serpulids), which are incapable of dominating space through competition. On the other hand, large substrates had increased probability of being colonized by a good competitor species (ascidians or sponges) that eventually would dominate space.
At the shores studied, large boulders were more frequently dominated, usually by bryozoans, serpulids and bivalves, groups that are bad competitors when faced with ascidians or sponges (Kay \& Keough, 1981; Russ, 1982). Keough (1984) also observed the dominance of bryozoans on boulders up to $180 \mathrm{~cm}^{2}$, equivalent size to the large boulders at São Sebastiao. According to him, ascidians and sponges dominated only substrata over $625 \mathrm{~cm}^{2}$. The apparent incongruence with results reported by Osman (1977) is probably because they were obtained on fouling plates that were not subjected to disturbance as were the small boulders, so that succession could proceed.

The intertidal region level was another important cause of the distribution of the fauna. The emersion and submersion of the habitat by the tides is a predictable kind of disturbance to which organisms can adapt themselves (Sousa, 1984). At São Sebastiao Channel, the medium intertidal region is emerged twice a day, usually during daytime, while the low intertidal region is not always emerged twice a day, and the lower tides occur usually at dawn or dusk times. On both shores, the most abundant organisms in the medium intertidal region had some kind of protection against desiccation such as the calcareous tubes of serpulids and the shells of bivalves. In the low intertidal region, bryozoans were also very abundant, and are relatively well protected against desiccation by the chitinous or calcareous test. The medium intertidal region had more sand than the low intertidal region on both shores, so that the abrasion of sand could have also disfavoured species without protection (ascidians and sponges).

\section{Conclusions}

Concluding, both the boulders size and the tide level influenced the distribution of the encrusting community on their undersurfaces. At Ponta do Baleeiro, species richness increased with boulders size because of random sampling, while the Intermediate Disturbance Hypothesis explains the species-area relationship at Praia Grande. Boulders in the medium intertidal region had very low values of diversity and fauna cover, which comprised mainly serpulids and bivalves. On the other hand, boulders in the low intertidal region had high values of diversity and fauna cover, mainly of bryozoans, but ascidians and sponges were also present in this level.

\section{Acknowledgements}

I would like to thank my advisor $\operatorname{Dr}$ A. Cecília Z. Amaral and Dr Sergio de Almeida Rodrigues for valuable discussion and stimulus during the whole work. Dr E. 
Hajdu, Dr G. Muricy and Dr B. Motte identified the sponges, A. E. Migotto identified the cnidarians, Dr J.-L. d'Hondt identified the bryozoans, and Dr C. Monniot and Dr F. Monniot help me with the identification of ascidians, to all them I am indebted. Dr S. A. Rodrigues, Dr C. M. Jacobi, and an anonimous reviewer read early drafts of this manuscript and gave improving suggestions. Centro de Biologia Marinha, University of São Paulo offered its facilities for field work. This work was sponsored by CNPq and CAPES. This paper is part of my PhD Thesis, which was developed at Universidade Estadual de Campinas.

\section{References}

COLEMAN, B. D.; MARES, M. A.; WILLIG, M. R. \& HSIEH, Y.-H. 1982. Randomness, area, and species richness. Ecology, 63(4):1121-1133.

CONNELL, J. H. 1978. Diversity in tropical rain forests and coral reefs. Science, 199(4335):1302-1310.

CONNOR, E. F. \& McCOY, E. D. 1979. The statistics and biology of the species-area relationship. Am. Naturalist, 13(6):791-833.

GUERAZZI, M. C. 1987. Estudos preliminares sobre a estrutura de uma comunidade de costão rochoso, em mesolitoral, num gradiente de salinidade. In: SIMPÓSIO SOBRE ECOSSISTEMAS DA COSTA SUL E SUDESTE BRASILEIRAS - SÍNTESE DOS CONHECIMENTOS. Cananéia, 1987. São Paulo, ACIESP, 54(2):221-232.

JACKSON, J. B. C. 1977. Habitat area, colonization, and development of epi-benthic community structure. In: Keegan, B. F.; Ceidigh, P. O. \& Boaden, P. J. S. eds Biology of Benthic Organisms. Pergamon Press Inc., Elmsford, NY, p. 349-358.

JOHNSCHER-FORNASARO, G.; LOPES, C. F. \& MILANELLII, J. C. C. 1990 . Similaridade faunística entre comunidades da zona entremarés de costōes rochosos do litoral norte do Estado de São Paulo. In: SIMPÓSIO DE ECOSSISTEMAS DA COSTA SUL E SUDESTE BRASILEIRA: ESTRUTURA E MANEJO, 2. Águas de Lindóia, 1987. São Paulo, ACIESP, 71(3):75-88.
KADEKARU, N.; QUARENTEI-SOUSA, R. C. A; ROQUETI-HUMAYTA, M. H. \& JOHNSCHER-FORNASARO, G. 1987. Estudo da fauna das comunidades bentônicas de substrato consolidado do litoral norte do Estado de São Paulo. II: Praia do Estaleiro. In: SIMPÓSIOSOBRE ECOSSISTEMAS DA COSTA SUL E SUDESTE BRASILEIRA - SÍNTESE DOS CONHECIMENTOS. Cananéia, 1987. São Paulo, ACIESP, 54(2):201-220.

KAY, A. M. \& KEOUGH, M. J. 1981. Occupation of patches in the epifaunal communities on pier pilings and the bivalve Pinna bicolor ao Edithburg, South Australia. Oecologia, 48:123-130.

KEEN, S. L. \& NEILL, W. E. 1980 . Spatial relationships and some structuring processes in benthic intertidal animal communities. J. expl mar. Biol. Ecol., 45:139-155.

KEOUGH, M. J. 1984. Effects of patch size on the abundance of sessile marine invertebrates. Ecology, 65(2):423-437.

LITTLER, M. M. \& LITTLER, D. S. 1981. Intertidal macrophyte communities from Pacific Baja California and the upper Gulf of California: relative constant vs. environmentally fluctuating systems. Mar. Ecol.-Prog. Ser., 4:145-158.

\& 1984.

Relationship between macroalgal functional form groups and substrata stability in a subtropical rocky-intertidal system. J. expl mar. Biol. Ecol., 74:13-34.

MacARTHUR, R. H. \& WILSON, E. O. 1963. An equilibrium theory of insular biogeography. Evolution, 17:373-387.

\& 1967. The Theory of Island Biogeography, Monographs in Population Biology. Princeton University Press, Princeton, s.p.

MAGURRAN, A. E. 1988. Ecological Diversity and its Measurement. Princeton University Press, Princeton, $179 \mathrm{p}$.

McGUINNESS, K. A. 1984. Species-area relations of communities on intertidal boulders: testing the null hypothesis. J. Biogeogr., 11:439-456. 
McGUINNESS, K. A. 1987a. Disturbance and organisms on boulders. I. Patterns in the environment and the community. Oecologia, 71(3):409-419.

1987b. Disturbance and organisms on boulders. II. Causes of patterns in diversity and abundance. Oecologia 71(3):420-430.

\& UNDERWOOD, A. J. 1986.

Habitat structure and the nature of communities on intertidal boulders. J. expl mar. Biol. Ecol., 104:97-123.

NALESSO, R. C. 1988. Influência da salinidade e exposição ao ar na distribuição dos mexilhões Brachidontes darwinianus e $B$. solisianus em dois estuários do litoral do Estado de São Paulo. Dissertaçāo de mestrado. Universidade Estadual de Campinas, Instituto de Biologia. 165 p.

NONATO, E. \& PÉRES, J. M. 1961. Observations sur quelques peuplements intertidaux de substrat dur dans la région d'Ubatuba (État S. Paulo). Cah. Biol. mar., 2:263-270.

OLIVEIRA FILHO, E. C. \& MAYAL, E. M. 1976. Seasonal distribution of intertidal organisms at Ubatuba, São Paulo (Brazil). Rev. bras. Biol., 36(2):305-316.

OSMAN, R. W. 1977. The establishment and development of a marine epifaunal community. Ecol. Monogr., 47:37-63.

1982. Artificial substrates as ecological islands. In: Cairns, J. ed. Artificial Substrates. Ann Arbor Science Publ., Ann Arbor, Michigan, p. 71-114.

PAULA, E. J. 1987. Zonação nos costôes rochosos: região entremarés. In: SIMPÓSIO SOBRE A COSTA SUL E SUDESTE BRASILEIRAS SÍNTESE DOS CONHECIMENTOS. Cananéia, 1987. São Paulo, ACIESP, 54(1):266-288.

PETERSEN, J. A.; SUTHERLAND, J. P. \& ORTEGA. S. 1986. Patch dynamics of mussel beds near Sâo Scbastião (São Paulo), Brazil. Mar. Biol., 93:389-393.

ROCHA, R. M. 1993. Comunidade incrustante em substrato duro não estabilizado na zona entremarés (SãoSebastião, SP). Tese de doutorado. Universidade Estadual de Campinas, Instituto de Biologia. 148 p.
ROCHA, R. M. 1994. Variabilidade do recrutamento larval na zona entremarés. In: SIMPÓSIO SOBRE ECOSSISTEMAS DA COSTA BRASILEIRA, 3. Anais. São Paulo, ACIESP, 87(3):301-308.

\& MONNIOT, F. 1993. Didemnum rodriguesi $\mathrm{sp}$ nov., a new didemnidae tunicate common to Southern Brazil and New Caledonia. Ann. Inst. océanogr., Paris., 69(2):261-265.

\& Some Didemnum

(Ascidiacea, Didemnidae) species in South-eastern Brazil. Revta bras. Biol. (in press).

RODRIGUES, S. A. \& SHIMIZU, G. Y. 1988. Ophionereis reticulata e Petrolisthes armatus: disputa pelo abrigo físico na baixa-mar? Ciência Cult., S Paulo, 40(7):691-692.

ROSSO, S. 1990. Estrutura de comunidades intermareais de substrato consolidado das proximidades da Baía de Santos (Sāo Paulo, Brasil): uma abordagem descritiva enfatizando aspectos metodológicos. Tese de doutorado. Universidade de São Paulo, Instituto de Biociências, 216 p.

RUSS, G. R. 1982. Overgrowth in a marine epifaunal community: competitive hierarchies and competitive networks. Oecologia, 53(1):12-19.

SCHOENER, A. 1974. Experimental zoogeography: colonization of marine mini-islands. Am. Naturalist, 108(964):715-738.

SHIMIZU, G. Y. \& RODRIGUES, S. A. 1988. Comunidade de fauna vágil de substrato arenoso sob pedras, da zona entremarés do Istmo do Baleeiro, São Sebastião (SP). Ciência Cult., S Paulo, 40(7):689-690.

SIMBERLOFF, D. 1976. Experimental zoogeography of islands: effects of island size. Ecology, 57(4):629-648.

SOUSA, W. P. 1979a. Experimental investigations of disturbance and ecological succession in a rocky intertidal algal community. Ecol. Monogr., 49(3):227-254.

1979b. Disturbance un marine boulder fields: the nonequilibrium maintenance of species diversity. Ecology, 60(6):1225-1239. 
SOUSA, W. P. 1984. The role of disturbance in natural communities. Ann. Rev. Ecol. Syst., 15:353-391.

TRALDI, E. S. \& SCHLENZ, E. 1990. Estratégias de ocupação de um costão rochoso por cinco espécies de anêmonas no litoral sul do Estado de São Paulo. In: SIMPÓSIO DE ECOSSISTEMAS DA COSTA SUL E SUDESTE BRASILEIRA: ESTRUTURA, FUNÇĀO E MANEJO, 2. Águas de Lindóia, 1990. São Paulo, ACIESP, 71(3):67-74.

(Manuscript received 24 May 1994; revised 17 July 1995; accepted 30 August 1995) 